\title{
A Limiting Transition in a Singularly Perturbed Equation with the Loss of Stability
}

\author{
Dilmurat Abdillajanovich Tursunov \\ Department of Informatics, Osh State University, Osh, Kyrgyzstan
}

Email address:

dosh2012@mail.ru

\section{To cite this article:}

Dilmurat Abdillajanovich Tursunov. A Limiting Transition in a Singularly Perturbed Equation with the Loss of Stability. International Journal of Theoretical and Applied Mathematics. Vol. 3, No. 1, 2017, pp. 43-48. doi: 10.11648/j.ijtam.20170301.17

Received: November 24, 2016; Accepted: January 3, 2017; Published: January 16, 2017

\begin{abstract}
A limiting transition is performed in some systems of singularly perturbed differential equations in the case of change of stability. This phenomenon is found in laser physics, chemical kinetics, plastic deformation, biophysics, in the modified Zieglers system, and in the simulation of upland forest fires, safe combustion with maximum temperature, etc. Cases when such equations have explicit solutions are extremely rare. For sufficiently small values of the parameter to determine the behavior of the solution a daunting task even for super computers, but it is possible with the asymptotic series. Therefore, studies of singularly perturbed problems when the condition of asymptotic stability is relevant.
\end{abstract}

Keywords: Singularly Perturbed, Cauchy Problem, Asymptotic Stability, Limited Equation, Solutions Asymptotic, Analytic Continue, Turning Point

\section{Introduction and Preliminaries}

The main aim of the study is to: Prove the asymptotic proximity of solutions of a singularly perturbed problem to the solution of the limit equation, in the case of change of stability. In a specific example is to prove that the whole range of unstable solutions of the Cauchy problem for a system of singularly perturbed ordinary differential equations with a turning point is asymptotically close to a solution of the limit system of equations. See the power of the method of analytic continuation.

The methods used in the study: conversion method, method of successive approximations, analytic continuation, stationary phase method.

The results: A new class of singularly perturbed ordinary differential equations with a turning point is identified. In this class the stability of the solution is stored in an unstable range. Passing to the limit in the case of change of stability is proved. We prove the asymptotic proximity of solutions of a singularly perturbed problem to the solution of the reduced equation on the interval $\left[t_{0},+\infty\right)$. Phenomenon, the delay of loss of stability in singularly perturbed systems, is found only in analytical systems.

In applications macrokinetics [1], plastic deformation [2], a modified system of the Ziegler [2], safe combustion with a maximum temperature [4-6] are common differential equations with a small parameter in the highest derivatives. The basis of such equations laid A. N. Tikhonov. He was the first in 1952 to formulate sufficient conditions under which the solutions are asymptotically close singularly perturbed problem and the limit equation [7]. Proven A. N. Tikhonov's theorem is called the "theorem on passing to the limit." Among the conditions in this theorem there is a condition that is associated with the asymptotic stability of the rest point, called the adjoint system. Researchers interested in the question, what will be the asymptotic behavior of solutions of the perturbed problem in violation of the conditions of stability.

In 1973, Shishkov M. A. for the first time in the particular example proved the possibility of passing to the limit, and if the condition of asymptotic stability [8]. The development of this approach is reflected in the papers [9-16].

In publications [12-15], the delay time of flow of the integral curves in violation of the conditions of asymptotic stability of course. In [16] first proved that the delay time of the integral curves of the flow when the condition of asymptotic stability can be endless. Continuing research in this area, we found a class of equations in which the interval 
of instability as it "disappears", i.e. throughout this unstable range occurs limit. In this paper, we present a concrete example of this class of equations, and prove the asymptotic proximity of solutions of a singularly perturbed problem to the solution of the limit equation on the interval $[-a / 2,+\infty)$.

\section{Statement of the Problem}

Consider the problem

$$
\begin{aligned}
& \varepsilon x^{\prime}(t, \varepsilon)=A(t) x(t, \varepsilon)+\varepsilon^{\alpha} f(t), \\
& x\left(t_{0}, \varepsilon\right)=x^{0}(\varepsilon),\left\|x^{0}(\varepsilon)\right\|<c \varepsilon,
\end{aligned}
$$

where $A(\mathrm{t})$ is a square matrix function of second order with elements $a_{j m}(t), f(t)=\left(f_{1}(t) f_{2}(t)\right)^{\mathrm{T}}, a_{j m}(t), f_{m}(t) j, m=1,2$ are analytic functions in $D$ ( $D$ is defined below), $1 / 2<\alpha \in \mathrm{R}, x_{0}(\varepsilon)$ $=\left(x_{1}{ }^{0}(\varepsilon) x_{2}{ }^{0}(\varepsilon)\right)^{\mathrm{T}}, t \in\left[t_{0},+\infty\right), t \in \mathrm{R}, c>0$ is arbitrarily const.

Condition 1. Let $A(t)$ is matrix function of the second order, has a complex conjugate eigenvalues $\lambda_{1,2}(t)=(a-t)(t \pm i b), 0<a$, $\sqrt{3} b \geq a, t_{0}=a / 2$.

The system (1) can be considered as a perturbation in relation to the degenerate system

$$
A(t) x^{*}(t)=0
$$

Degenerate system (3) has the trivial solution $x^{*}(t)=0$.

For the eigenvalues $\lambda_{1,2}(t)$ function $A(t)$ satisfies the inequalities:

$$
\begin{gathered}
\operatorname{Re}\left(\lambda_{1,2}(t)\right)=-t(t-a)<0, \text { for } t \in(-\infty, 0) \cup(a,+\infty) ; \\
\operatorname{Re}\left(\lambda_{1,2}(t)\right)=-t(t-a)>0 \text { for } t \in(0, a) ; \\
\operatorname{Re}\left(\lambda_{1,2}(t)\right)=-t(t-a)=0 \text { for } t=0, t=a .
\end{gathered}
$$

In the terminology of $\mathrm{W}$. Wasow or M. V. Fedoryuk point $t$ $=a$ is called a turning point, as $\lambda_{1}(a)=\lambda_{2}(a)$.

Definition 1. The interval where the inequality $\operatorname{Re}\left(\lambda_{1,2}(t)\right)<0$, we call sustainable. A interval, for which the condition $\operatorname{Re}\left(\lambda_{1,2}(t)\right)>0$, is called unstable interval.

Definition 2. The point $t=t^{*}$ is called the transition point, if the real part of the eigenvalues changes sign when passing through this point.

In the present case $t \in(-\infty, 0) \cup(a,+\infty)$ is steady interval; $t \in(0, a)$ is intermittent interval; $t=0, t=a$ is transition point.

In the stable range of all the conditions of Theorem A. N. Tikhonov passing to the limit, and therefore the equality:

$$
x(t, \varepsilon)=x *(t), t \in\left[t_{0}, 0\right),
$$

i.e., solution of the perturbed problem is asymptotically close to the solution of the limiting equation. As noted above, in the unstable range remains an open question.

The novelty of this work is to prove the equality $\boldsymbol{x}(t, \varepsilon)=\boldsymbol{x}^{*}(t)=0$ for $t \in\left[t_{0},+\infty\right) \supset[0, a]$.

To bring the $A(t)$ diagonalized perform the following conversion $B^{-1}(t) A(t) B(t)=\Lambda(t)$, where

$$
\begin{gathered}
A(t)=\left(\begin{array}{ll}
a_{11}(t) & a_{12}(t) \\
a_{21}(t) & a_{22}(t)
\end{array}\right), \mathrm{B}(t)= \\
\left(\begin{array}{cc}
\lambda_{1}(t)-a_{22}(t) & \lambda_{2}(t)-a_{22}(t) \\
a_{21}(t) & a_{21}(t)
\end{array}\right), \Lambda(t)=\operatorname{diag}\left(\lambda_{1}(t), \lambda_{2}(t)\right) .
\end{gathered}
$$

Suppose that in $D$ the inequality $\operatorname{det} B(t) \neq 0$. This condition is satisfied, for example if

$$
\begin{gathered}
\mathrm{A}(t)=\left(\begin{array}{cc}
-t(t-a) & b(t-a) \\
-b(t-a) & -t(t-a)
\end{array}\right), \text { then } \mathrm{B}(t)=\left(\begin{array}{cc}
i & -i \\
1 & 1
\end{array}\right), \text { det } \\
B(t)=2 i \neq 0 .
\end{gathered}
$$

Equation (1) using the transformation $x(t, \varepsilon)=B(t) y(t, \varepsilon)$ brought to the form

$$
\begin{gathered}
\varepsilon y^{\prime}(t, \varepsilon)=\Lambda(t) y(t, \varepsilon)+\varepsilon^{\alpha} h(t)+\varepsilon g(t) y(t, \varepsilon), \\
y\left(t_{0}, \varepsilon\right)=y^{0}(\varepsilon),\left\|y^{0}(\varepsilon)\right\|=\mathrm{O}(\varepsilon),
\end{gathered}
$$

where $h(t)=B^{-1}(t) f(t), g(t)=-B^{-1}(t) B^{\prime}(t)$.

The Cauchy problem for differential equations (4)-(5) we replace the integral equation:

$$
y(t, \varepsilon)=E\left(t, t_{0}, \varepsilon\right) y^{0}+\int_{t_{0}}^{t} E(t, \tau, \varepsilon)\left(g(\tau) y(\tau, \varepsilon)+\varepsilon^{\alpha-1} h(\tau)\right) d \tau
$$

where $E(t, \tau, \varepsilon)=\exp \left(\int_{\tau}^{t} \Lambda(s) d s / \varepsilon\right)$.

To solve the integral equation (6) apply the method of successive approximations:

$$
\begin{gathered}
\text { Let } y_{0}(t, \varepsilon) \equiv 0 \\
y_{n}(t, \varepsilon)=E\left(t, t_{0}, \varepsilon\right) y^{0}(\varepsilon)+\int_{t_{0}}^{t} E(t, \tau, \varepsilon)\left(g(\tau) y_{n-1}(\tau, \varepsilon)+\varepsilon^{\alpha-1} h(\tau)\right) d \tau
\end{gathered}
$$

then

$$
\begin{gathered}
y_{1}(t, \varepsilon)=E\left(t, t_{0}, \varepsilon\right) y^{0}(\varepsilon)+\varepsilon^{\alpha-1} \int_{t_{0}}^{t} E(t, \tau, \varepsilon) h(\tau) d \tau, \\
y_{n}(t, \varepsilon)=y_{1}(t, \varepsilon)+\int_{t_{0}}^{t} E(t, \tau, \varepsilon) g(\tau) y_{n-1}(\tau, \varepsilon) d \tau .
\end{gathered}
$$

\section{Main Results}

Estimation successive approximations. In assessing the successive approximations $\left\{y_{\mathrm{n}}(t, \varepsilon)\right\}$ real variable $t$, we assume complex variable $t=t_{1}+i t_{2}, t_{1}, t_{2} \in \mathrm{R}, i=\sqrt{-1}$.

$$
\begin{gathered}
y_{1}=E\left(t_{1}, t_{2}, t_{0}, \varepsilon\right) y^{0}(\varepsilon)+\varepsilon^{\alpha-1} \int_{t_{0}}^{t_{1}+i t_{2}} E\left(t_{1}, t_{2}, \tau_{1}, \tau_{2}, \varepsilon\right) h\left(\tau_{1}, \tau_{2}\right) d\left(\tau_{1}+i \tau_{2}\right) . \\
y_{n}=y_{1}+\int_{t_{0}}^{t_{1}+i t_{2}} E\left(t_{1}, t_{2}, \tau_{1}, \tau_{2}, \varepsilon\right) g\left(\tau_{1}, \tau_{2}\right) y_{n-1}\left(\tau_{1}, \tau_{2}, \varepsilon\right) d\left(\tau_{1}+i \tau_{2}\right) .
\end{gathered}
$$


Let $y_{n}(t, \varepsilon)=\left(y_{1, \mathrm{n}}(t, \varepsilon) y_{2, \mathrm{n}}(t, \varepsilon)\right)^{\mathrm{T}}, h(t)=\left(h_{1}(t) h_{2}(t)\right)^{\mathrm{T}}, g(t)=\left\{\mathrm{g}_{\mathrm{j} m}(t)\right\}$, $j, m=1,2$.

Then

$$
\begin{gathered}
y_{j, 1}=O(\varepsilon) e^{\frac{u_{j}\left(t_{1}, t_{2}\right)}{\varepsilon}}+\varepsilon^{\alpha-1} \int_{t_{0}}^{t_{1}+i t_{2}} h_{j}\left(\tau_{1}, \tau_{2}\right) e^{\frac{u_{j}\left(t_{1}, t_{2}\right)-u_{j}\left(\tau_{1}, \tau_{2}\right)}{\varepsilon}} d\left(\tau_{1}+i \tau_{2}\right), \\
y_{j, m}=y_{j, 1}+\int_{t_{0}}^{t_{1}+i t_{2}}\left(g_{j 1} y_{1, m-1}+g_{j 2} y_{2, m-1}\right) e^{\frac{u_{j}\left(t_{1}, t_{2}\right)-u_{j}\left(\tau_{1}, \tau_{2}\right)}{\varepsilon}} d\left(\tau_{1}+i \tau_{2}\right) \\
j=1,2 ; m>1, \quad u_{j}\left(t_{1}, t_{2}\right)=\int_{t_{0}}^{t_{1}+i t_{2}} \lambda_{j}(s) d s .
\end{gathered}
$$

Asymptotic estimates for the functions $y_{j, m}\left(t_{1}, t_{2}, \varepsilon\right) j=1,2$, $m \in \mathrm{N}$ calculate in $D: D=D_{1} \cup D_{2}$, where $D_{1}=\left\{\left(t_{1}, t_{2}\right)\right.$ : $\left.\operatorname{Re}\left(u_{j}\left(t_{1}, t_{2}\right)\right) \leq 0, t_{0} \leq t_{1} \leq a, j=1,2\right\}$,

$$
D_{2}=\left\{\left(\mathrm{t}_{1}, 0\right): a<t_{1}<+\infty\right\} .
$$

Note that $\left[t_{0},+\infty\right) \subset D$ at $t_{2} \neq 0$ and $\left[t_{0},+\infty\right)=D$ at $t_{2}=0$. Consider now the function

$$
u_{j}\left(t_{1}, t_{2}\right)=\int_{t_{0}}^{t_{1}+i t_{2}} \lambda_{j}(s) d s, j=1,2, t_{0}=-a / 2:
$$

$u_{1}\left(t_{1}, t_{2}\right)=u_{11}\left(t_{1}, t_{2}\right)+i u_{12}\left(t_{1}, t_{2}\right), u_{2}\left(t_{1}, t_{2}\right)=u_{21}\left(t_{1}, t_{2}\right)+i u_{22}\left(t_{1}, t_{2}\right)$,

where $u_{11}\left(t_{1}, t_{2}\right)=-\left(t_{1}-a\right)^{3} / 3-a\left(t_{1}-a\right)^{2} / 2+\left(t_{1}-a / 2\right) t_{2}^{2}-b t_{2}\left(t_{1}-a\right)$,

$$
\begin{gathered}
u_{12}\left(t_{1}, t_{2}\right)=t_{2}^{3} / 3-t_{2}^{2} b / 2-\left(t_{1}-a\right)^{2}\left(b / 2-t_{2}\right)-a t_{2}\left(t_{1}-a\right), \\
u_{21}\left(t_{1}, t_{2}\right)=u_{11}\left(t_{1},-t_{2}\right), u_{22}\left(t_{1}, t_{2}\right)=u_{12}\left(t_{1},-t_{2}\right) .
\end{gathered}
$$

Getting Started to the construction area $D_{1}$. Since $u_{11}\left(t_{1}, t_{2}\right)=u_{21}\left(t_{1},-t_{2}\right)$, so that lines $u_{11}\left(t_{1}, t_{2}\right)=0$ and $u_{21}\left(t_{1}, t_{2}\right)=0$ are symmetric about the real axis.

From the equation $u_{11}\left(t_{1}, t_{2}\right)=0$, we have:

$$
\begin{aligned}
& \left(t_{1}-a / 2\right) t_{2}^{2}-k\left(t_{1}-a\right) t_{2}-\left(t_{1}-a\right)^{3} / 3-a\left(t_{1}-a\right)^{2} / 2=0, \\
& \text { or } \quad\left(\left(t_{1}-a / 2\right) t_{2}-\varphi_{1}\left(t_{1}, a, b\right) / 2\right)\left(t_{2}-\varphi_{2}\left(t_{1}, a, b\right)\right)=0,
\end{aligned}
$$$$
\text { where } \varphi_{1}\left(t_{1}, a, b\right)=\left(t_{1}-a\right)\left(b+\sqrt{b^{2}-a^{2} / 3+4 t_{1}^{2} / 3}\right) \text {, }
$$

$$
\varphi_{2}\left(t_{1}, a, b\right)=\left(t_{1}-a\right)\left(b-\sqrt{b^{2}-a^{2} / 3+4 t_{1}^{2} / 3}\right) /\left(2 t_{1}-a\right),
$$

By the condition 1 , holds $0<a, b \geq a / \sqrt{3}$, so $b^{2}-a^{2} / 3+4 t_{1}^{2}$ $/ 3 \geq 0$. Separately consider the cases: 1) $b>a / \sqrt{3}$;2) $b=a / \sqrt{3}$.

I. Let $b>a / \sqrt{3}$, whereas smooth line $\varphi_{2}\left(t_{1}, a, b\right)$ connecting point $\left(t_{0}, 0\right)$ and $(a, 0)$.

Construct graphs of implicit functions $u_{11}\left(t_{1}, t_{2}\right)=0$ and $u_{21}\left(t_{1}, t_{2}\right)=0$ using the package Maple:

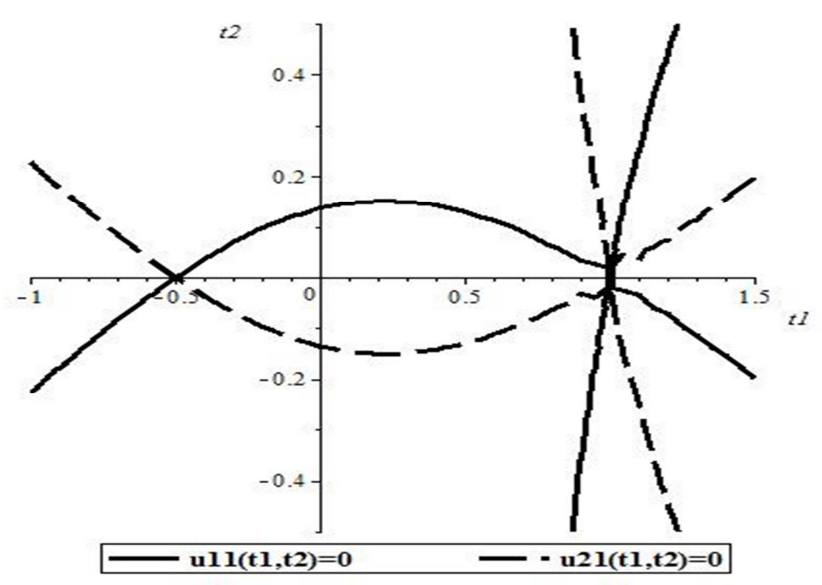

Figure 1. Case $\sqrt{3} b>a$, for $a=1, b=2$.

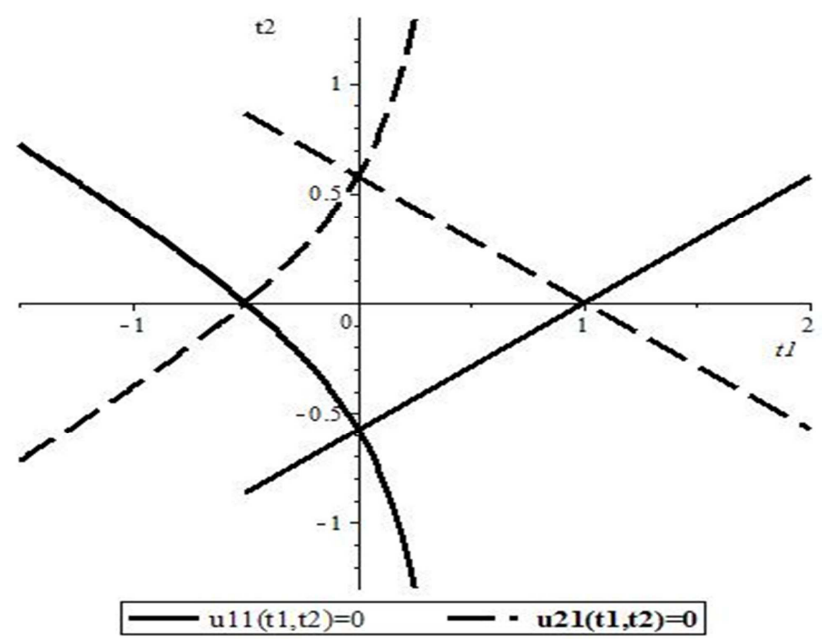

Figure 2. Case $\sqrt{3} b=a$, for $a=1$.

In the neighborhood of the point $(a, 0)$ function $u_{11}\left(t_{1}, t_{2}\right)$ $\left(u_{21}\left(t_{1}, t_{2}\right)\right)$ divides the plane into four equal sectors in which signs of the functions $u_{11}\left(t_{1}, t_{2}\right), u_{21}\left(t_{1}, t_{2}\right)$ alternates. Both functions are negative in the sector, which contains the real axis $\mathrm{O} t_{1}$. From the intersection of the domains $u_{11}\left(t_{1}, t_{2}\right) \leq 0$ and $u_{21}\left(t_{1}, t_{2}\right) \leq 0$ ranging $t_{0} \leq t_{1} \leq a$ we obtain the desired region $D_{1}$. Consequently, the boundary area $D_{1}$ (figure 1) is composed of smooth line $t_{2}=-\varphi_{2}\left(t_{1}, a, b\right)$ and $t_{2}=\varphi_{2}\left(t_{1}, a, b\right)$, connecting points $\left(t_{0}, 0\right)$ and $(a, 0)$.

We proceed to the estimation the functions $y_{j, 1}\left(t_{1}, t_{2}, \varepsilon\right)$ in $D$. For this we first compute the integrals:

$$
J_{j}\left(t_{1}, t_{2}, \varepsilon\right)=\int_{L_{j}} h_{j}\left(\tau_{1}, \tau_{2}\right) e^{\frac{u_{j}\left(t_{1}, t_{2}\right)-u_{j}\left(\tau_{1}, \tau_{2}\right)}{\varepsilon}} d\left(\tau_{1}+i \tau_{2}\right), j=1,2 .
$$

If $\left(t_{1}, t_{2}\right) \in D_{1}$, then $J_{1}\left(t_{1}, t_{2}, \varepsilon\right)$ integration path $\mathrm{L}_{1}=\mathrm{L}_{11} \cup \mathrm{L}_{12}$, where $\mathrm{L}_{11}$ is the line $\tau_{2}=\varphi_{2}\left(\tau_{1}, a, b\right)$ at $\mathrm{t}_{0} \leq \tau_{1} \leq t_{1} ; \mathrm{L}_{12}$ is the line $\tau_{1}=t_{1}$ when $t_{2}^{*} \geq \tau_{2} \geq t_{2}, t_{2}^{*}=\varphi_{2}\left(t_{1}, a, b\right)$. Symmetrical line $\mathrm{L}_{2}$ to $\mathrm{L}_{1}$ with respect to the real axis will determine the path of integration for $J_{2}\left(t_{1}, t_{2}, \varepsilon\right)$.

We proceed to the calculation of integrals:

$$
J_{j}\left(t_{1}, t_{2}, \varepsilon\right)=j_{j 1}\left(t_{1}, t_{2}, \varepsilon\right)+j_{j 2}\left(t_{1}, t_{2}, \varepsilon\right), j=1,2
$$




$$
\begin{aligned}
& \text { where } j_{11}\left(t_{1}, t_{2}, \varepsilon\right)=\int_{t_{0}}^{t_{1}} \tilde{h}_{1}\left(\tau_{1}, \phi_{2}\left(\tau_{1}, a, k\right)\right) e^{\tilde{u}_{1}\left(t_{1}, t_{2}, \tau_{1}\right) / \varepsilon} d \tau_{1} \text {, } \\
& \tilde{h}_{1}\left(\tau_{1}, \phi_{2}\left(\tau_{1}, a, b\right)\right)=h_{1}\left(\tau_{1}, \phi_{2}\left(\tau_{1}, a, b\right)\right)\left(1+i \phi_{2}^{\prime}\left(\tau_{1}, a, b\right)\right), \\
& \tilde{u}_{1}\left(t_{1}, t_{2}, \tau_{1}\right)=u_{1}\left(t_{1}, t_{2}\right)-u_{1}\left(\tau_{1}, \phi_{2}\left(\tau_{1}, a, b\right)\right) \\
& j_{12}\left(t_{1}, t_{2}, \varepsilon\right)=i \int_{t_{2}^{*}}^{t_{2}} h_{1}\left(t_{1}, \tau_{2}\right) e^{\left(u_{1}\left(t_{1}, t_{2}\right)-u_{1}\left(t_{1}, \tau_{2}\right)\right) / \varepsilon} d \tau_{2} \\
& j_{21}\left(t_{1}, t_{2}, \varepsilon\right)=\int_{t_{0}}^{t_{1}} \tilde{h}_{2}\left(\tau_{1}, \phi_{2}\left(\tau_{1}, a, k\right)\right) e^{\tilde{u}_{2}\left(t_{1}, t_{2}, \tau_{1}\right) / \varepsilon} d \tau_{1}, \\
& \tilde{h}_{2}\left(\tau_{1}, \phi_{2}\left(\tau_{1}, a, b\right)\right)=h_{2}\left(\tau_{1},-\phi_{2}\left(\tau_{1}, a, b\right)\right)\left(1-i \phi_{2}^{\prime}\left(\tau_{1}, a, b\right)\right) \text {, } \\
& \tilde{u}_{2}\left(t_{1}, t_{2}, \tau_{1}\right)=u_{2}\left(t_{1}, t_{2}\right)-u_{2}\left(\tau_{1},-\phi_{2}\left(\tau_{1}, a, b\right)\right) \\
& j_{22}\left(t_{1}, t_{2}, \varepsilon\right)=i \int_{-t_{2}^{*}}^{t_{2}} h_{2}\left(t_{1}, \tau_{2}\right) e^{\left(u_{2}\left(t_{1}, t_{2}\right)-u_{2}\left(t_{1}, \tau_{2}\right)\right) / \varepsilon} d \tau_{2} \\
& u_{1}\left(\tau_{1}, \varphi_{2}\left(\tau_{1}, a, b\right)\right)=i\left(\tau_{1}-a\right)^{2} p_{1}\left(\tau_{1}, a, b\right), \\
& p_{1}\left(\tau_{1}, a, b\right)=\frac{\left(9 b^{2}+a^{2}\right)^{2}}{162 b^{3}}+O\left(\left(\tau_{1}-\frac{a}{2}\right)\right) \text {; }
\end{aligned}
$$

$\operatorname{Re}\left(u_{1}\left(t_{1}, t_{2}\right)-u_{1}\left(t_{1}, \tau_{2}\right)\right)=\left(t_{2}-\tau_{2}\right)\left(\left(t_{1}-a / 2\right)\left(t_{2}+\tau_{2}\right)-b\left(t_{1}-a\right)\right)=$

$$
=\left(t_{2}-\tau_{2}\right)\left(\left(2 t_{1}-a\right) \zeta+b\left(a-t_{1}\right)\right)<0, \zeta \in\left(t_{2}, \tau_{2}\right) ;
$$$$
u_{2}\left(\tau_{1},-\varphi_{2}\left(\tau_{1}, a, b\right)\right)=u_{1}\left(\tau_{1}, \varphi_{2}\left(\tau_{1}, a, b\right)\right)=i\left(\tau_{1}-a\right)^{2} p_{1}\left(\tau_{1}, a, b\right) \text {; }
$$$$
\operatorname{Re}\left(u_{2}\left(t_{1}, t_{2}\right)-u_{2}\left(t_{1}, \tau_{2}\right)\right)=\left(t_{2}-\tau_{2}\right)\left(\left(t_{1}-a / 2\right)\left(t_{2}+\tau_{2}\right)+b\left(t_{1}-a\right)\right)=
$$

$$
=\left(t_{2}-\tau_{2}\right)\left(\left(2 t_{1}-a\right) \zeta_{1}-b\left(a-t_{1}\right)\right)<0, \zeta_{1} \in\left(\tau_{2}, t_{2}\right) .
$$

Applying the method of stationary phase [17] for $J_{j}\left(t_{1}, t_{2}, \varepsilon\right)$ in $D$ we obtain the following asymptotic estimate:

$$
\begin{aligned}
& J_{1}\left(t_{1}, t_{2}, \varepsilon\right) \sim \mathrm{O}(\sqrt{\varepsilon}) H_{1}(a)+\mathrm{O}(\varepsilon), \\
& J_{2}\left(t_{1}, t_{2}, \varepsilon\right) \sim \mathrm{O}(\sqrt{\varepsilon}) H_{2}(a)+\mathrm{O}(\varepsilon),
\end{aligned}
$$

where $H_{1}\left(t_{1}\right)=h_{1}\left(t_{1}, \varphi_{2}\left(t_{1}, a, b\right)\right)\left(1+\frac{\partial \phi_{2}\left(t_{1}, a, b\right)}{\partial t_{1}}\right)$,

$$
H_{2}\left(t_{1}\right)=h_{2}\left(t_{1},-\varphi_{2}\left(t_{1}, a, b\right)\right)\left(1-\frac{\partial \phi_{2}\left(t_{1}, a, b\right)}{\partial t_{1}}\right) .
$$

From here

$$
J_{1}\left(t_{1}, t_{2}, \varepsilon\right) \sim \mathrm{O}(\sqrt{\varepsilon}), J_{2}\left(t_{1}, t_{2}, \varepsilon\right) \sim \mathrm{O}(\sqrt{\varepsilon})
$$

If $\left(t_{1}, t_{2}\right) \in D_{2}$, then $\mathrm{L}_{1}=\mathrm{L}_{11} \cup \mathrm{L}_{12}$, where $\mathrm{L}_{11}$ is the line $\tau_{2}=\varphi_{2}\left(\tau_{1}, a, k\right)$ with $\mathrm{t}_{0} \leq \tau_{1} \leq a ; \mathrm{L}_{12}$ is the line $\tau_{2}=0$ for $a \leq \tau_{1} \leq \mathrm{t}_{1}$. Similarly, the symmetric line $\mathrm{L}_{2}$ to $\mathrm{L}_{1}$ with respect to the real axis is obtained by integrating for $J_{2}\left(t_{1}, t_{2}, \varepsilon\right)$. We have:

$$
J_{j}\left(t_{1}, t_{2}, \varepsilon\right)=\tilde{j}_{j 1}\left(t_{1}, t_{2}, \varepsilon\right)+\tilde{j}_{j 2}\left(t_{1}, 0, \varepsilon\right), j=1,2
$$

where $\tilde{j}_{11}\left(t_{1}, t_{2}, \varepsilon\right)=\int_{t_{0}}^{a} \tilde{h}_{1}\left(\tau_{1}, \phi_{2}\left(\tau_{1}, a, b\right)\right) e^{\tilde{u}_{1}\left(t_{1}, t_{2}, \tau_{1}\right) / \varepsilon} d \tau_{1}$

$$
\begin{gathered}
\tilde{j}_{12}\left(t_{1}, 0, \varepsilon\right)=\int_{a}^{t_{1}} h_{1}\left(\tau_{1}, 0\right) e^{\left(u_{1}\left(t_{1}, 0\right)-u_{1}\left(\tau_{1}, 0\right)\right) / \varepsilon} d \tau_{1} ; \\
j_{21}\left(t_{1}, t_{2}, \varepsilon\right)=\int_{t_{0}}^{a} \tilde{h}_{2}\left(\tau_{1}, \phi_{2}\left(\tau_{1}, a, b\right)\right) e^{\tilde{u}_{2}\left(t_{1}, t_{2}, \tau_{1}\right) / \varepsilon} d \tau_{1} ; \\
j_{22}\left(t_{1}, 0, \varepsilon\right)=\int_{a}^{t_{1}} h_{2}\left(\tau_{1}, 0\right) e^{\left(u_{2}\left(t_{1}, 0\right)-u_{2}\left(\tau_{1}, 0\right)\right) / \varepsilon} d \tau_{1} .
\end{gathered}
$$

Consequently, we obtain exactly the same estimate (7).

II. Let $b=a / \sqrt{3}$, then we have: $\varphi_{1}\left(t_{1}, a, b\right)=\left(t_{1}-a\right)\left(a+2 t_{1}\right) / \sqrt{3}$,

$$
\begin{gathered}
\varphi_{2}\left(t_{1}, a, b\right)=-\left(t_{1}-a\right) / \sqrt{3}, \text { i.e. } \\
u_{11}\left(t_{1}, t_{2}\right)=\left(\left(t_{1}-a / 2\right) t_{2}-\varphi_{1}\left(t_{1}, a, b\right) / 2\right)\left(t_{2}-\varphi_{2}\left(t_{1}, a, b\right)\right)= \\
=\left(\sqrt{3}\left(2 t_{1}-a\right) t_{2}-\left(t_{1}-a\right)\left(a+2 t_{1}\right)\right)\left(\sqrt{3} t_{2}+t_{1}-a\right) / 6, \\
u_{21}\left(t_{1}, t_{2}\right)=\left(\sqrt{3}\left(2 t_{1}-a\right) t_{2}+\left(t_{1}-a\right)\left(a+2 t_{1}\right)\right)\left(\sqrt{3} t_{2}-t_{1}+a\right) / 6 .
\end{gathered}
$$

In the neighborhood of the points $(a, 0)$ and $(0, b)$ function $u_{11}\left(t_{1}, t_{2}\right)$ divides the plane into four equal sectors in which the sign of the function $u_{11}\left(t_{1}, t_{2}\right)$ alternates. Similarly, the function $u_{21}\left(t_{1}, t_{2}\right)$ in the neighborhood of the points $(a, 0)$ and $(0,-b)$ divides the plane into four equal sectors in which the sign of the functions $u_{21}\left(t_{1}, t_{2}\right)$ alternates. Both functions are negative in the sector, which contains the real axis $\mathrm{Ot}_{1}$. From the intersection of the domains $u_{11}\left(t_{1}, t_{2}\right) \leq 0$ and $u_{21}\left(t_{1}, t_{2}\right) \leq 0$ ranging $t_{0} \leq t_{1} \leq a$ we obtain the desired region $D_{1}$. Consequently, the boundary area $D_{1}$ (figure 2) consists of a line:

$$
\begin{gathered}
t_{2}= \pm\left(t_{1}-a\right)\left(a+2 t_{1}\right) /\left(\sqrt{3}\left(2 t_{1}-a\right)\right) \text { at } t_{0} \leq t_{1} \leq 0 \text { and } \\
t_{2}= \pm\left(t_{1}-a\right) / \sqrt{3} \text { at } 0 \leq t_{1} \leq a .
\end{gathered}
$$

Graphs of implicit functions $u_{11}\left(t_{1}, t_{2}\right)=0$ and $u_{21}\left(t_{1}, t_{2}\right)=0$ are constructed in the system Maple. For the integrals:

$$
J_{j}\left(t_{1}, t_{2}, \varepsilon\right)=\int_{L_{j}} h_{j}\left(\tau_{1}, \tau_{2}\right) e^{\frac{u_{j}\left(t_{1}, t_{2}\right)-u_{j}\left(\tau_{1}, \tau_{2}\right)}{\varepsilon}} d\left(\tau_{1}+i \tau_{2}\right)
$$

define the path of integration:

If $\left(t_{1}, t_{2}\right) \in D_{1}$, then $J_{1}\left(t_{1}, t_{2}, \varepsilon\right)$ integration path $\mathrm{L}_{1}=\mathrm{L}_{11} \cup \mathrm{L}_{12}$, where $\mathrm{L}_{11}$ is the line $\tau_{2}=\psi_{1}\left(\tau_{1}, a\right)=\left(\tau_{1}-a\right)\left(a+2 \tau_{1}\right) /\left(\sqrt{3}\left(2 \tau_{1}-a\right)\right)$ when $t_{0} \leq \tau_{1} \leq 0 ; \mathrm{L}_{12}$ is the line $\tau_{2}=\psi_{2}\left(\tau_{1}, t_{1}, t_{2}, k\right)=k+\left(t_{2}-k\right) \tau_{1} / t_{1}$ when $0 \leq \tau_{1} \leq t_{1}$. Symmetrical line $\mathrm{L}_{2}$ to $\mathrm{L}_{1}$ with respect to the real axis is obtained by integrating for $J_{2}\left(t_{1}, t_{2}, \varepsilon\right)$. We have: 


$$
J_{j}\left(t_{1}, t_{2}, \varepsilon\right)=j_{j 3}\left(t_{1}, t_{2}, \varepsilon\right)+j_{j 4}\left(t_{1}, t_{2}, \varepsilon\right), j=1,2
$$

where $j_{13}=\int_{t_{0}}^{0} \tilde{h}_{1}\left(\tau_{1}, \psi_{1}\left(\tau_{1}, a\right)\right) e^{\left(u_{1}\left(t_{1}, t_{2}\right)-u_{1}\left(\tau_{1}, \psi_{1}\left(\tau_{1}, a\right)\right)\right) / \varepsilon} d \tau_{1}$

$$
\begin{gathered}
j_{14}=\int_{0}^{t_{1}} \tilde{h}_{1}\left(\tau_{1}, \psi_{2}\left(\tau_{1}, t_{1}, t_{2}, k\right)\right) e^{\left(u_{1}\left(t_{1}, t_{2}\right)-u_{1}\left(t_{1}, \psi_{2}\left(\tau_{1}, t_{1}, t_{2}, k\right)\right)\right) / \varepsilon} d \tau_{1} ; \\
j_{23}=\int_{t_{0}}^{0} \tilde{h}_{2}\left(\tau_{1},-\psi_{1}\left(\tau_{1}, a\right)\right) e^{\left(u_{2}\left(t_{1}, t_{2}\right)-u_{2}\left(\tau_{1},-\psi_{1}\left(\tau_{1}, a\right)\right)\right) / \varepsilon} d \tau_{1} ; \\
j_{24}=\int_{0}^{t_{1}} \tilde{h}_{2}\left(\tau_{1},-\psi_{2}\left(\tau_{1}, t_{1}, t_{2}, k\right)\right) e^{\left(u_{2}\left(t_{1}, t_{2}\right)-u_{2}\left(t_{1},-\psi_{2}\left(\tau_{1}, t_{1}, t_{2}, k\right)\right)\right) / \varepsilon} d \tau_{1} ; \\
u_{1}\left(\tau_{1}, \psi_{1}\left(\tau_{1}, a\right)\right)=i 4\left(\tau_{1}-a\right)^{2}\left(a^{2}-2 \tau_{1} a+4\left(\tau_{1}\right)^{2}\right)^{2} /\left(9 \sqrt{3}\left(a-2 \tau_{1}\right)^{3}\right), \\
u_{2}\left(\tau_{1},-\psi_{2}\left(\tau_{1}, t_{1}, t_{2}, k\right)\right)=u_{1}\left(\tau_{1}, \psi_{2}\left(\tau_{1}, t_{1}, t_{2}, k\right)\right) .
\end{gathered}
$$

Evaluating the integrals, we obtain exactly the same estimate (7).

If $\left(t_{1}, t_{2}\right) \in D_{2}$, then for $J_{1}\left(t_{1}, t_{2}, \varepsilon\right)$ integration path $\mathrm{L}_{1}=\mathrm{L}_{11} \cup \mathrm{L}_{12} \cup \mathrm{L}_{13}$, where $\mathrm{L}_{11}$ is the line $\tau_{2}=\left(\tau_{1}-a\right)\left(a+2 \tau_{1}\right) /(\sqrt{3}$ $\left.\left(2 \tau_{1}-a\right)\right)$ at $t_{0} \leq \tau_{1} \leq 0 ; \mathrm{L}_{12}$ is the line $\tau_{2}=\psi_{2}\left(\tau_{1}, a, 0, b\right)=-\left(\tau_{1}-a\right) /$ $\sqrt{3}$ at $0 \leq \tau_{1} \leq a ; \quad L_{13}$ is the line $\tau_{2}=0$ for $a \leq \tau_{1} \leq t_{1}$. Here, similarly, the symmetric line $\mathrm{L}_{2}$ to $\mathrm{L}_{1}$ with respect to the real axis is obtained by integrating for $J_{2}\left(t_{1}, t_{2}, \varepsilon\right)$.

$$
J_{j}\left(t_{1}, t_{2}, \varepsilon\right)=j_{j 3}\left(t_{1}, t_{2}, \varepsilon\right)+j_{j 5}\left(t_{1}, t_{2}, \varepsilon\right)+\tilde{j}_{j 2}\left(t_{1}, t_{2}, \varepsilon\right), j=1,2 ;
$$

where $j_{j 3}\left(t_{1}, t_{2}, \varepsilon\right), \quad \tilde{j}_{j 2}\left(t_{1}, t_{2}, \varepsilon\right), j=1,2$ are as defined above;

$$
\begin{gathered}
j_{25}\left(t_{1}, t_{2}, \varepsilon\right)=\int_{0}^{t_{1}} \tilde{h}_{2}\left(\tau_{1}\right) e^{\tilde{u}_{2}\left(t_{1}, t_{2}, \tau_{1}\right) / \varepsilon} d \tau_{1} \\
\tilde{u}_{2}\left(t_{1}, t_{2}, \tau_{1}\right)=u_{2}\left(t_{1}, t_{2}\right)-u_{2}\left(t_{1},-\psi_{2}\left(\tau_{1}, a, 0, b\right)\right), \\
\left.\tilde{h}_{2}\left(\tau_{1}\right)=h_{2}\left(\tau_{1},-\psi_{2}\left(\tau_{1}, a, 0, b\right)\right)\left(1-i \psi_{2}^{\prime}\left(\tau_{1}, a, 0, b\right)\right)\right) ; \\
j_{15}\left(t_{1}, t_{2}, \varepsilon\right)=\int_{0}^{t_{1}} \tilde{h}_{1}\left(\tau_{1}\right) e^{\tilde{u}_{1}\left(t_{1}, t_{2}, \tau_{1}\right) / \varepsilon} d \tau_{1}, \\
\tilde{u}_{1}\left(t_{1}, t_{2}, \tau_{1}\right)=u_{1}\left(t_{1}, t_{2}\right)-u_{1}\left(t_{1}, \psi_{2}\left(\tau_{1}, a, 0, b\right)\right), \\
\left.\tilde{h}_{1}\left(\tau_{1}\right)=h_{1}\left(\tau_{1}, \psi_{2}\left(\tau_{1}, a, 0, b\right)\right)\left(1+i \psi_{2}^{\prime}\left(\tau_{1}, a, 0, b\right)\right)\right) ; \\
u_{1}\left(\tau_{1}, \psi_{2}\left(\tau_{1}, a, 0, b\right)\right)=i 4\left(\tau_{1}-a\right)\left(a+2 \tau_{1}\right) / 9 \sqrt{3}, \\
u_{2}\left(\tau_{1},-\psi_{2}\left(\tau_{1}, a, 0, b\right)\right)=u_{1}\left(\tau_{1}, \psi_{2}\left(\tau_{1}, a, 0, b\right)\right) .
\end{gathered}
$$

Applying the method of stationary phase, we obtain a similar asymptotic estimates (7).

As a result, we obtain asymptotic estimates for the first approximation:

$$
y_{j, 1}\left(t_{1}, t_{2}, \varepsilon\right) \sim \mathrm{O}(\varepsilon)+\mathrm{O}\left(\varepsilon^{\alpha-1 / 2}\right) \sim \mathrm{O}\left(\varepsilon^{\alpha-1 / 2}\right), \alpha-1 / 2>0, j=1,2 .
$$

For the rest of successive approximations $y_{j, \mathrm{k}}\left(t_{1}, t_{2}, \varepsilon\right)$ we have:

$$
\begin{gathered}
y_{j, 2}\left(t_{1}, t_{2}, \varepsilon\right) \sim y_{j, 1}+\mathrm{O}(1)\left(y_{1,1}+y_{2,1}\right) J_{j, 1}\left(t_{1}, t_{2}, \varepsilon\right) ; \\
y_{j, 3}\left(t_{1}, t_{2}, \varepsilon\right) \sim y_{j, 1}+\mathrm{O}(1)\left(y_{1,2}+y_{2,2}\right) J_{j, 1}\left(t_{1}, t_{2}, \varepsilon\right) ; \\
\cdots \\
y_{j, \mathrm{n}}\left(t_{1}, t_{2}, \varepsilon\right) \sim y_{j, 1}+\mathrm{O}(1)\left(y_{1, \mathrm{n}-1}+y_{2, \mathrm{n}-1}\right) J_{j, 1}\left(t_{1}, t_{2}, \varepsilon\right) .
\end{gathered}
$$

From, we have

$$
\begin{gathered}
y_{j, 2}\left(t_{1}, t_{2}, \varepsilon\right) \sim \mathrm{O}\left(\varepsilon^{\alpha-1 / 2}\right)\left(1+\mathrm{O}\left(\varepsilon^{1 / 2}\right)\right) ; \\
y_{j, 3}\left(t_{1}, t_{2}, \varepsilon\right) \sim \mathrm{O}\left(\varepsilon^{\alpha-1 / 2}\right)\left(1+\mathrm{O}\left(\varepsilon^{1 / 2}\right)+\mathrm{O}(\varepsilon)\right) ; \\
\cdots \\
y_{j, \mathrm{n}}\left(t_{1}, t_{2}, \varepsilon\right) \sim \mathrm{O}\left(\varepsilon^{\alpha-1 / 2}\right)\left(1+\mathrm{O}\left(\varepsilon^{1 / 2}\right)+\mathrm{O}(\varepsilon)+\ldots+\mathrm{O}\left(\varepsilon^{(\mathrm{n}-1) / 2}\right)\right) \text { or } \\
y_{j, \mathrm{n}}\left(t_{1}, t_{2}, \varepsilon\right) \sim \mathrm{O}\left(\varepsilon^{\alpha-1 / 2}\right)\left(1-\mathrm{O}\left(\varepsilon^{\mathrm{n} / 2}\right)\right) /\left(1-\mathrm{O}\left(\varepsilon^{1 / 2}\right)\right) . \\
y_{j}\left(t_{1}, t_{2}, \varepsilon\right)=\lim _{n \rightarrow \infty} y_{j, \mathrm{n}}\left(t_{1}, t_{2}, \varepsilon\right)=\mathrm{O}\left(\varepsilon^{\alpha-1 / 2}\right) .
\end{gathered}
$$

Therefore, $\boldsymbol{x}(t, \varepsilon)=\mathrm{O}\left(\varepsilon^{\alpha-1 / 2}\right), \alpha>1 / 2$.

Correct is

Theorem. Let condition 1 be satisfied then, the problem (1)-(2) has a unique solution $\boldsymbol{x}(t, \varepsilon)$ which is satisfy the estimates

$$
\|x(t, \varepsilon)\| \leq c \varepsilon^{\beta}, \text { for } t \in\left[t_{0},+\infty\right) \supset[0, a],
$$

where $c>0$ is arbitrarily const and $\beta=\alpha-1 / 2>0$.

Hence, it follows that the equality

$$
\lim _{\varepsilon \rightarrow 0} x(t, \varepsilon)=x *(t)=0 \text { for } t \in\left[t_{0},+\infty\right) \supset[0, a] \text { is validity. }
$$

\section{Conclusion}

Built leading asymptotic term solutions of singularly perturbed systems of ordinary differential equations, when the complex conjugate eigenvalues are real and zeros in the complex plane. Zeros eigenvalues on the real axis referred to as the pivot point. The resulting asymptotic estimate for the solution of this problem is not improving. A specific example is proved that the entire range of unstable solution of the Cauchy problem for a system of singularly perturbed ordinary differential equations is asymptotically close to a solution of the limit system of equations. We prove a limit as inappropriate conditions for asymptotic stability. The asymptotic proximity of solutions of a singularly perturbed problem to the solution of the limit equation on the interval $\left[t_{0},+\infty\right)$.

The set of systems of differential equations with such eigenvalues (or similar) form a new class of singularly perturbed systems of ordinary differential equations in which the unstable interval does not affect the stability of the solution. A system of singularly perturbed differential 
equations with complex conjugate eigenvalues require further research.

\section{References}

[1] Sobolev V. A., Shhepakina E. A. Reduction models and critical phenomena in macrokinetics. Moscov, Fizmatlit. 2010. $320 \mathrm{p}$.

[2] Semenov M. E., Kolupaeva S. N., Rozhnov A. I. Mathematical modeling of plastic deformation of GCK materials under varying strain rate. Herald of Perm National Research Polytechnic University. Mechanics. 2011. №3. pp. 100-117.

[3] Neishtadt A. I. and Sidorenko V. V., Stability loss delay in a Ziegler system. J. App. MathsMechs. 1997. Vol. 61, No. 1, pp. 15-25.

[4] Golodova E. S., Shhepakina E. A. Modelling of safe combustion with a maximum temperature. Mathematical modeling. 2008. Vol. 20. No. 5. pp. 55-58.

[5] Golodova E. S., Shchepakina E. A. Maximal combustion temperature estimation. Journal of Physics: Conf. Series. 2006. V. 55. P. 94-104.

[6] Shhepakina E. A. Singular perturbations in the problem of safe combustion regimes. Mathematical modeling. 2003. Vol. 15. No. 8. pp. 113-117.

[7] Tihonov A. N. Systems of differential equations containing small parameters in the derivatives. Mat. compilation. Mathematics collection. 1952. 31 (73). no. 3. pp. 575-586.

[8] Shishkova, M. A. Consideration of a system of differential equations-tions with a small parameter in the highest derivatives. Dokl. USSR Academy of Sciences. 1973. vol. 209, no. 3. pp. 576-579.
[9] Nefedov N. N., Schneider K. R. On immediate-delayed exchange of stabilities and periodic forced canards. Journal of Computational Mathematics and Mathematical Physics. 2008. Vol. 48. No. 1. pp. 46-61.

[10] Tursunov D. A. Uniform asymptotic solutions of the Cauchy problem for a generalized model equation of L. S. Pontryagin in the case of violation of conditions of asymptotic stability. Science Journal of Applied Mathematics and Statistics. New York. 2013. Vol. 1, No. 3. pp. 25-29.

[11] Golodova E. S., Shhepakina E. A. Evaluation of delayed loss of stability in differential systems with trajectories-ducks. Bulletin of the Samara State University. Natural science series. 2013. No. (104). pp. 12-24.

[12] Karimov S. Asymptotic behavior of solutions of certain classes of differential equations with a small parameter in the derivatives in the case of change of stability in the plane of the rest point «quick movements». Dr. Diss. Osh, 1983. 260 p.

[13] Alybaev K. S. Method of level lines of research singularly perturbed equations in violation of conditions of stability. Dr. Diss. Zhalalabad, 2001. 203 p.

[14] Pankov P. S., Alybaev K. S., Tampagarov K. B., Narbaev K. B. The phenomenon of boundary-layer lines and the asymptotic behavior of solutions of singularly perturbed linear ordinary differential equations with analytic functions. Bulletin of the Osh state university. 2013. No. 1. pp. 227-231.

[15] Tursunov D. A. Asymptotics of solution of singularly perturbed problem with periodic turning points in complex plane. Bulletin of the Tomsk Polytechnic University. 2014. vol. 324. no. 2. pp. 40-46.

[16] Tursunov D. A. Asymptotic expansion solution of singular perturbed problems, when complex conjugation eigenvalues has $\mathrm{n}$ multiple poles. PhD diss. Osh, 2005. $110 \mathrm{p}$.

[17] Fedorjuk M. V. Saddle-point method. Moscov, Librokom Publ., 2010. 368 p. 